\title{
Primary User Signal Recognition Algorithm based on AL-ABM in Cognitive network
}

\author{
Wang Xin* \\ School of Information \& Control Engineering \\ Shenyang Jianzhu University \\ Shenyang, China \\ e-mail: arnold0110@sina.com \\ * Corresponding Author \\ Huang Kuan \\ School of Information \& Control Engineering \\ Shenyang Jianzhu University \\ Shenyang, China \\ Zhang Na \\ College of Art and Design \\ Shenyang Jianzhu University \\ Shenyang, China \\ Wang Shi \\ School of Information \& Control Engineering \\ Shenyang Jianzhu University \\ Shenyang, China \\ Wang Changtao \\ School of Information \& Control Engineering \\ Shenyang Jianzhu University \\ Shenyang, China
}

\author{
Wang Shuai \\ School of Information \& Control Engineering \\ Shenyang Jianzhu University \\ Shenyang, China \\ Wang Xiuhong \\ Library \\ Shenyang Jianzhu University \\ Shenyang, China \\ Gong Wei \\ School of Information \& Control Engineering \\ Shenyang Jianzhu University \\ Shenyang, China \\ Wang Bin \\ Northeastern University at Qinhuangdao \\ Qinhuangdao, China
}

\begin{abstract}
The cognitive users can use the spectrum holes which are vacant at some time or some frequency. Once the authorized users need to use the spectrum again, the cognitive users should stop using it to avoid interfering primary users. In this paper, a method based on active learning (AL) and AdaBoost machine (ABM) for the primary user signal modulation type recognition is proposed in low signal to noise ratio. A set of cyclic spectrum features are first calculated, and the training samples and testing samples are formed for classification. Then, active learning algorithm is applied to obtain samples improved classification through a number of iterations, and AdaBoost is formed. Finally, the formed SVM is utilized to recognize the primary user signal modulation type. The simulation results shows that the proposed AL-ABM Algorithm can improve the recognition accuracy of signal samples obviously.
\end{abstract}

Keywords-Cognitive network, Active Learning, Primary User Signal Recognition, AdaBoost Machine

\section{INTRODUCTION}

Recently radio spectrum resource has become increasingly scarce due to the development of communication. In order to improve the efficiency of the spectrum usage, cognitive radio is proposed and considered to be a promising technique to solve problems of increasing scarcity of spectrum $[1,2]$. The cognitive users can use the spectrum holes which are vacant at some time or some frequency. Once the authorized users need to use the spectrum again, the cognitive users should stop using it to avoid interfering primary users.

As one of the most important issues in cognitive radio networks[3,4], spectrum sensing is proposed to solve the shortage of vacant spectrum in recent. Spectrum sensing mainly includes follow three techniques: energy detection, cyclostationary feature detection and matched filtering[5]. In view of the radio channel complex environment, spectrum sensing technology focused on spectrum detection problem under low signal-to-noise ratio circumstances of channel multi-path fading and shadow fading. In [6], The authors proposed spectrum sensing algorithm based on the maximum - minimum eigenvalue (MME), but Spectrum sensing effect of the algorithm is not ideal, detection rate is low in the low SNR. The authors in [7] proposed spectrum sensing algorithm that using cyclic spectral energy(CSE) as the detecting statistic to obtain detection threshold, but due to an error cyclic spectrum estimation of eigenvalues, there was a shortage in testing performance in the low signal-to-noise ratio environment. The SVM, developed by Vapnik and others [8], has been used for many machine learning tasks such as pattern recognition and object classifications. A SVM- 
based spectrum sensing algorithms proposed in [9] was applied to the field of cognitive radio, but SVM algorithm affects the classification effect directly due to the high computational complexity, to large-scale training samples, prone to overfitting and other reasons.In [10], a novel signal separation algorithm for spectrum sensing and signal separation which based on wavelet transform and blind source separation. In [11,12] a reliable scheme for cooperative spectrum sensing based on double threshold energy detection and Dempster-Shafer (D-S) theory was proposed.

In this article, spectrum sensing is studied from the machine learning and an primary user signal recognition method based on AL-ABM is proposed. A set of received signal spectrum features are extracted the important feature vector as training samples and testing samples for classification. Then, active learning algorithm is applied to obtain samples improved classification. Finally, we utilize the trained $A B M$ to recognize the primary user signal modulation type.

The remainder of the paper is organized as follows. In section 2, the system model is described. The extraction of characteristic parameters is introduced in section 3 . The proposed algorithm is investigated in section 4 and is well validated with computer simulation in section 5 . The several concluding remarks are made in section 6 .

\section{THE SYSTEM MODEL}

In this paper, we assume that a cognitive radio network with $\mathrm{W}$ primary users and $\mathrm{M}$ secondary users, for any one of the secondary user, the presence of the primary user can be summarized as a hypothesis test model of two elements

$$
\left\{\begin{array}{c}
H_{0}: y(t)=n(t) \\
H_{1}: y(t)=\sum_{w=1}^{W} s_{w}(t)+n(t)
\end{array}\right.
$$

Where $H_{0}$ denotes that the primary user is not exist, $H_{l}$ denotes that the primary user exists. A process $s(t)$, that is any one of primary users, is said to be cyclostationary in building indoor environment if its mean and variance are periodic with a period $T ; n(t)$ represents the additive white Gauss noise, the mean is zero, variance is $\sigma_{n}^{2}$.

On the basis of this model, We have extracted the cyclic spectrum characteristic parameters of the presence of the primary user or not, and obtain characteristic vector as adaboost machine training samples, thus we generate adaboost machine and spectrum sensing model. As we see from figure 1 .



Figure 1. spectrum sensing model

\section{SPECTRAL CORRELATION ANALYSIS AND SignAL FEATURE EXTRACTION}

Many signals in communication system exhibit cyclostationarity property called spectral correlation features, these property can be used for signal detection and recognition..

The spectral correlation function (SCF)of the signal $y(t)$ is obtained from the Fourier transform of the cyclic autocorrelation in Equation (2).

$$
S^{\alpha}(f)=\int_{-\infty}^{\infty} R^{\alpha}(\tau) \exp (-2 \pi \alpha t j) d t
$$

Because the discrete Fourier transform $Y(k)$ of received signal $y(t)$ is Gauss process $y(t)$, s weighted linear combination, is still a Gauss process. The cyclic spectrum $S(k)$ is consist of $L$ independent and identically distributed random variables, according to the central limit theorem, $S(k)$ obeys the Gauss distribution under $L \square 1$.

Based on the calculation of the spectral correlation function, we can obtain three parameters as characteristic parameters of a signal modulation identification. The first one consists of the largest value of all spectral correlation function. The second feature to extract is the correlation coefficient for the SCF. The third feature of the set is the spectrum energy $\varepsilon$.

1. The largest value of all spectral correlation function

We assume that $S(k)$ is the largest value of all spectral correlation function $S^{\alpha}(f)$ as the first extracted characteristic parameter.

2. The correlation coefficient for the SCF

The correlation coefficient for the SCF between frequency components $f \pm 2 \alpha$ can be calculated as

$$
C_{y}^{\alpha}(f)=\frac{S^{\alpha}(f)}{[S(f+\alpha / 2) S(f-\alpha / 2)]^{\frac{1}{2}}}
$$

\section{The spectrum energy $\varepsilon$}

The largest energy spectrum is the spectrum energy $\varepsilon$, which is defined as

$$
E=\frac{1}{K} \sum_{k=0}^{K-1}|S(k)|^{2}
$$

The above characteristic parameters are formed characteristic vector as the input samples.

In this section, a novel approach to recognize the primary user signal modulation type is introduced based on the proposed system model.

We have extracted the cyclic spectrum characteristic parameters of the presence of the primary user or not $\left(a_{1}^{q}, a_{2}^{q}, \cdots, a_{M}^{q}\right)$, and obtain characteristic vector $y_{q}=\left(a_{1}^{q}, a_{2}^{q}, \cdots, a_{M}^{q}\right)^{T}, q=1,2, \cdots, M$ as ABM training samples, thus we generate $\mathrm{ABM}$ and using it to test input samples. 


\section{SPeCtrum Sensing Algorithm BaSEd ON ACTIVE LEARNING AND ADABOOST}

In this section, a novel approach to spectrum sensing is introduced based on the proposed system model. AL-ABM algorithm can be expressed as follow.

1. A few samples selected randomly from sampleset Uare marked manual tags and constitutes a training sample set L.

2. Training samples are randomly selected from the training set $\mathrm{L}$ to train the classifier, then establishing the initial classifer.
3.According to the standard Q query, A number of samples of the never marked samples in sample sets $U$ are selected and annotated, then placed in a training set $\mathrm{L}$.

4. Active learning classifier are re-training until the classifier reachs the final trainingstandards.

5. Repeat above steps, the primary user signal modulation type can be recognized by utilizing trained active learning.

AdaBoost algorithm can be expressed as figure1.



Figure 2. Adaboost Algorithm

\section{SimUlation AND ANALYSIS}

In order to verify that this algorithm in low signal-tonoise ratio environment quality, using Matlab7.0 to complete experiments for MSK, BPSK and AM. The simulations are carried out with 1000 samples at SNR ranging from $-15 \mathrm{~dB}$ to $5 \mathrm{~dB}$, the length of DFT cyclic spectrum is 512 .

Table 1 is for both cases of signal-to-noise ratio for the $-5 \mathrm{~dB}$ and $-15 \mathrm{~dB}$, using MME, ANN and the article arithmetic on 3 types modulation classification of signals from the correct rate. Simulation results show that MME, ANN and this algorithm detects the correct rate have decreased for modulated signals when the signal-to-noise ratio gradually reduced. When the signal to noise ratio $\mathrm{S} / \mathrm{N}=-15 \mathrm{~dB}$, AM signal detection algorithms in this article the right rates were higher than both ANN and MME algorithms with $12.5 \%$ and $63.8 \%$, for MSK signal, this algorithm the algorithm detects the correct rate is significantly higher than the other two algorithms.

Figure 3 shows the accuracy rate of the different algorithms for MSK, it is observed that the three algorithms have better detection accuracy rate. The accuracy decreases with decreases of signal-to-noise ratio, the accuracy rate of MME algorithm is $8.6 \%$, ANN is $59.8 \%$, the proposed algorithm accuracy rate is $80.0 \%$ in $\mathrm{S} / \mathrm{N}=-15 \mathrm{~dB}$. Above results show that detection performance of the proposed algorithm is significantly higher than the other two algorithms, which demonstrates the feasibility of our algorithm. 
TABLE I ACCURACY RATE OF THE DIFFERENT ALGORITHMS FOR PRIMARY USER SIGNAL RECOGNIT ION

\begin{tabular}{|c|c|c|c|c|c|c|}
\hline \multirow{2}{*}{$\begin{array}{c}\text { Signal } \\
\text { Types }\end{array}$} & \multicolumn{2}{|c|}{$\begin{array}{c}\text { Correct rate\% (S/N=- } \\
15 \mathrm{~dB})\end{array}$} & \multicolumn{2}{|c|}{ Correct rate\% (S/N=-5dB) } \\
\cline { 2 - 7 } & MME & ANN & $\begin{array}{c}\text { Our } \\
\text { Best }\end{array}$ & MME & ANN & Our Best \\
\hline AM & 18.3 & 69.6 & 82.1 & 82.3 & 85.4 & 93.2 \\
\hline BPSK & 12.1 & 62.3 & 78.5 & 78.5 & 84.2 & 88.5 \\
\hline MSK & 8.6 & 59.8 & 80.0 & 76.6 & 83.0 & 91.1 \\
\hline
\end{tabular}



Fig 3. the accuracy rate of the different algorithms for MSK

\section{CONCLUSIONS}

This paper focuses on the primary user signal detection issue in low SNR environment, presented primary user signal recognition method based on AL-ABM.The algorithm utilizes spectral correlation analysis in signal feature extraction and AdaBoost algorithm to treat primary user signal detection. Simulation results show that this algorithm can better improve the spectrum sensing performance of the primary user signal in low signal-tonoise ratio environment.

\section{ACKNOWLEDGMENT}

This work was financially supported by Program for New Century Excellent Talents in University (NCET-120103).

\section{REFERENCES}

[1] Haykin, S., Thomson, D., and Reed, J. “Spectrum sensing for cognitive radio” . Proc. IEEE, May 2009, vol. 97, no. 5, pp. 849877.

[2] Devroye, N., Vu, M., and Tarokh, V., "Cognitive radio networks". IEEE Signal Processing Mag., Nov. 2008, vol. 25, pp. 12-23.

[3] Yucek, T., and Arslan, H., "A survey of spectrum sensing algorithms for cognitive radio applications" .IEEE Commun. Surveys Tutorials, quarter. 2009, vol. 11, no.1, pp. 116-130.

[4] Wang, B., and Liu, K. J. R. "Advances in cognitive radio networks: A survey”. IEEE J. Sel. Topics Signal Process, Feb. 2011,vol. 5, no. 1 , pp. 5-23.

[5] Wang X., Wang J.K., Liu Z.G. Spectrum sensing algorithm based on random forest in cognitive network. Chinese Journal of Scientific Instrument. 2013, Vol. 34, no. 11, pp. 35-41.

[6] Ghasemi, A., and Sousa, E., "Spectrum sensing in cognitive radio networks: requirements, challenges and design trade-offs". IEEE Commun. Mag, Apr. 2008, vol. 46, no. 4, pp. 32-39.

[7] BHARGAVI, D., and MURTHY, C. R. "Performance comparison of energy, matched-filter and cyclostationarity based spectrum sensing".2010 IEEE Eleventh International Workshop on Signal Processing Advances in Wireless Communications (SPAWC). 2010. pp.1-5.

[8] ZENG Y H, LIANG Y C. "Eigenvalue-based spectrum sensing algorithms for cognitive radio". IEEE Transactions on Communications, 2009, vol. 57, no. 6, pp.1784-1793.

[9] He, A., Bae, K. K., and Newman, T., "A survey of artificial intelligence for cognitive radios". IEEE Trans. Veh. Technol., May 2010, vol. 59, no. 4, pp. 1578-1592.

[10] Dong, X., Li, Y., and Wu, C. "A learner based on neural network for cognitive radio". in 12th IEEE International Conference on Communication Technology (ICCT '10), Nanjing, China, Nov 2010, pp. 893-896.

[11] Cortes,C. and Vapnik, V. "Support-vector networks" . Machine Learning, 1995, 20, pp.273-297.

[12] Hu, H., Song,J., and Wang, Y. "Signal classification based on spectral correlation analysis and SVM in cognitive radio". in Advanced Information Networking and Applications, 2008. AINA 2008. 22th International Conference on, Mar 2008, pp. 883-887. 